\title{
PHYLOGENETIC TREE ON LERNAEOSIS IN ARWANA FISH (Scleropages jardinii)
}

\author{
Dikry Novel Shatrie*\#, Kurniasih ${ }^{* *}$, Nurcahyo, W.**), and Triyanto**) \\ ") Centre for Fish Quarantine, Ministry of Marine Affair and Fisheries, Jakarta \\ ${ }^{* *}$ Faculty of Veterinary Medicine Gadjah Mada University, Jogjakarta
}

(Received 4 January 2011 ; Accepted 1 May 2011 )

\begin{abstract}
Arwana Irian fish is one of the endangered species. Some studies on arwana Irian fish found that Lernaeosis attacked arwana Irian fish. Lernaeosis is one of the diseases that cause the high mortality in juvenile fish. The objectives of this research was to find out the species of Lernaea (Copepoda) often attacked arwana Irian fish. Lernaea sp. was collected from Papua and Jakarta (Java). They were fixed in the ethanol absolute solution for DNA sequencing in 28S DNA region with primer 28SF (5'-ACA ACT GTG ATC CCC TTA G-3'); 28SR (5' TGG TCC GTG TTT CAA GAC G-3'). It was found five different species of Lernaea and one of them was thought as a new species, based on the morphology. However, based on the phylogenetic analysis, they showed three different groups. Lernaea cyprinacea G., L. papuensis, L. devastatrix, and L. lophiara were in one group; L. cyprinacaea and L. oryzophila were in one groups; and the new Lernaea $\mathrm{sp}$. was in the different group.
\end{abstract}

\section{KEYWORDS: Scleropages jardinii, Lernaeosis, Phylogenetic tree}

\section{INTRODUCTION}

Lernaeid copepods cause serious deleterious effects on their freshwater fish hosts (Kabata, 1985). One factor which may contribute to the increased infestation level during the drying phase was the reduction in water volume. The economic loss caused by lernaeid ectoparasites has increased due to numerous epizootics occurring among the most important farmed fish in various parts of the world (Tasawar et al., 2007). A lernaeid population found only on jaws of Tilapia (Oreochromis spp.) Lernaea victoria (Fryer, 1961) has been identified as Lernaea cyprinacea. Other lernaeids of African fish were endemic, Lernaea barnimiana may be found on both cyprinid and cichlid hosts (Paperna, 1996). The embed- ded anchors were surrounded by fibrous granulation tissue, and there was considerable leucocytic response below the dermis. Myofibril degeneration and hemorrhage were noted in most sections of fish (Berry et al., 1991). Lernaea minuta was found in Javanese carp (Puntius gonionotus), which inhibated in Selangor, West Malaysia (Kularatne et al., 1994). Lernaea cyprinacea was reported from several fish such as goldfish (Carassius auratus L.), Helostoma temmincki, Cyprinus carpio, Carassius auratus L. (Shariff et al., 1986). Infestations of Lernaea cyprinacea was found on four native fish species (Galaxias occidentalis Ogilby; Edelia vittata Castelnau; Tandanus bostocki Whitley) and three introduced fish species (Carassius auratus L.; Gambusia holbrooki (Girard); Phalloceros

\# Corresponding author. Centre for Fish Quarantine, Ministry of Marine Affair and Fisheries, Jl. Medan Merdeka Timur No. 16, Jakarta 101 10, Indonesia. Tel.: +62 213519070

E-mail address:dikry_shatrie@yahoo.com 
caudimaculatus (Hensel) (Marina et al., 2008). The phylogenetic relationships among the Ergasilidae, included Lernaea cyprinacea from China, were examined using neighbor-joining, maximum parsimony, maximum likelihood, and Bayesian inference methods based on partial sequences of $18 \mathrm{~S}$ and $28 \mathrm{~S}$ ribosomal deoxyribonucleic acid, respectively (Song et al., 2008). However, the molecular sequence analysis and phylogenetic relationships among the parasitic Lernaea sp. still unclear. Here we would like to provide additional resolution for the interpretation of Lernaea sp., in addition to use identification based on morphology. The objective of this paper is to identify Lernaea sp. based on molecular analysis and to find phylogenetic relationships among 6 spesies of Lernaea.

\section{MATERIALS AND METHODS}

A total of 20 of parasitic copepods belonging to Lernaeidae were collected from arwana fish in Papua, Indonesia. Copepods were examined under stereo microscope to identify species. Specimens were fixed in ethanol absolute for molecular study. Primer was used, 28SR(5'-TGG TCC GTG TTT CAA GAC G-3') and 28SF (5'-ACA ACT GTG ATG CCC TTA G-3'); 28SR (5' TGG TCC GTG TIT CAA GAC G-3').

Following conditions of cycles were $94^{\circ} \mathrm{C}$ for 5 minutes, followed by 30 cycles of $94^{\circ} \mathrm{C}$ for 30 second, $54^{\circ} \mathrm{C}$ for 30 second and $72^{\circ} \mathrm{C}$ for 1 minute with a final extension at $72^{\circ} \mathrm{C}$ for 5 minutes (Song et al., 2008). Six specimens of Lernaea were then purified and sequenced in the $28 \mathrm{~S}$ region.

Results of DNA sequencing were sorted using Clustal W, continued with limited editing manually (Thompson et al., 1994). The distances between pairs were corrected with Kimura two-parameter model using MEGA 4 (Kumar et al., 2004). All sequences were aligned with Clustal W. Maximum parsimony (MP) and Neighbor joining (NJ) methods using 1,000 bootstrap re-sampling were used to get the phylogenetic tree.

\section{RESULTS AND DISCUSSIONS}

The sequense results showed that there were several gene variation between Lernaea lophiara (L1), L. papuensis ( $L 10)$, and $L$. devastatrix (L11) in one group; Lernaea cyprinacaea (L2) dan L. oryzophilla (L3) were in one group; and Lernaea sp. (L4) was in one group (Figure 1). The sequences result of Lernaea in Indonesia has not been reported yet.

From a total of 16 sequences at $28 \mathrm{~S}$ rDNA region where 430 is variable from 754 of parsimony, including gaps. And 289 is the Converse of 754 including the gap (Table 1).

Phylogenetic tree using maximum parsimony (MP) analysis found three groups among

L 1: AGCACTGA -CCGCCAGCTTTTGAAAGGGTTGCGCGGAATGTAGTGTTTG

L 2 : AGCACTGAAC- GCCAGCTTTTGAAAGGGTTGCGCGGAATGTAGTGTTTG

L 3: AGCACTGAAC- GCCAGCTTTTGAAAGGGTTGCGCGGAATGTAGTGTTTG

L 4: AGCACTGAACCGCCAGCTTTTGAAAGGGTTGCGCGGAATGTAGTGTTTG

L10: AGCACTGA - CCGCCAGCTTTTGAAAGGGTTGCGCGGAATGTAGTGTTTG

L11: AGCCTGGA - CCGCCAGCTTTTGAAAGGGTTGCGCGGAATGTAGTGTTTG

L 1: GGAGAGCCTTCTCATGATGCGCGGTGCAAAATCTGTCTAAGTCCACCTTG

L 2: GGAGAGCCTTCTCATGATGCGCGGTGCAAAATCTGTCTAAGTCCACCTTG

L 3 : GGAGAGCCTTCTCATGATGCGCGGTGCAAAATCTGTCTAAGTCCACCTTG

L 4: GGAGAGCCTTCTCATGATGCGCGGTGCAAAATCTGTCTAAGTCCACCTTG

L10: GGAGAGCCTTCTCATGATGCGCGGTGCAAAATCTGTCTAAGTCCACCTTG

L11: GGAGAGCCTTCTCATGATGCGCGGTGCAAAATCTGTCTAAGTCCACCTTG

L 1: ACTGGGGCCACTACCCATAGAGGGTGATAGGCCCGTAAGACAGTCTGCGT

L 2: ACTGGGGCCACTACCCATAGAGGGTGATAGGCCCGTAAGACAGTCTGCGT

L 3: ACTGGGGCCACTACCCATAGAGGGTGATAGGCCCGTAAGACAGTCTGCGT

L 4: ACTGGGGCCACTACCCATAGAGGGTGATAGGCCCGTAAGACAGTCTGCGT

L 10: ACTGGGGCCACTACCCATAGAGGGTGATAGGCCCGTAAGACAGTCTGCGT

L 11: ACTGGGGCCACTACCCATAGAGGGTGATAGGCCCGTAAGACAGTCTGCGT

Figure 1. Sequencing result of Lernaea species from arwana Irian fish 
Figure 1. (Continued)

L 1: GTtGTGCTGGCTTTTTCCCTAGAGTCGAGTTGCTTGGGAGTGCAGCTCAAA

L 2 : GTTGTGCTGGCTTTTTCCCTAGAGTCGAGTTGCTTGGGAGTGCAGCTCAAA

L 3 : GTTGTGCTGGCTTTTTCCCTAGAGTCGAGTTGCTTGGGAGTGCAGCTCAAA

L 4 : GTTGTGCTGGCTTTTTCCCTAGAGTCGAGTTGCTTGGGAGTGCAGCTCAAA

L10: GTTGTGCTGGCTTTTTCCCTAGAGTCGAGTTGCTTGGGAGTGCAGCTCAAA

L11: GTTGTGCTGGCTTTTTCCCTAGAGTCGAGTTGCTTGGGAGTGCAGCTCAAA

L 1: GTGCGTGGTAAACTCCACGTAAGGCTAAATATCACCCCGAGACCGATAGC L 2: GTGCGTGGTAAACTCCACGTAAGGCTAAATATCACCCCGAGACCGATAGC L 3: GTGCGTGGTAAACTCCACGTAAGGCTAAATATCACCCCGAGACCGATAGC L 4 : GTGCGTGGTAAACTCCACGTAAGGCTAAATATCACCCCGAGACCGATAGC L10: GTGCGTGGTAAACTCCACGTAAGGCTAAATATCACCCCGAGACCGATAGC L11: GTGCGTGGTAAACTCCACGTAAGGCTAAATATCACCCCGAGACCGATAGC

L 1: GAACAAGTACCGTGAGGGAAAGTTGAAAAGAACTTTGAAGAGAGAGTTCA L 2: GAACAAGTACCGTGAGGGAAAGTTGAAAAGAACTTTGAAGAGAGAGTTCA L 4: GAACAAGTACCGTGAGGGAAAGTTGAAAAGAACTTTGAAGAGAGAGTTCA L10: GAACAAGTACCGTGAGGGAAAGTTGAAAAGAACTTTGAAGAGAGAGTTCA L11: GAACAAGTACCGTGAGGGAAAGTTGAAAAGAACTTTGAAGAGAGAGTTCA

L 1 : ATAGTACGTGAAACTGTGTAGCGGTAAACAGAGGGGCTCTCGAAGTCCAG L 2: ATAGTACGTGAAACTGTGTAGCGGTAAACAGAGGGGCTCTCGAAGTCCAG L 3 : ATAGTACGTGAAACTGTGTAGCGGTAAACAGAGGGGCTCTCGAAGTCCAG L 4 : ATAGTACGTGAAACTGTGTAGCGGTAAACAGAGGGGCTCTCGAAGTCCAG L10: ATAGTACGTGAAACTGTGTAGCGGTAAACAGAGGGGCTCTCGAAGTCCAG L11: ATAGTACGTGAAACTGTGTAGCGGTAAACAGAGGGGCTCTCGAAGTCCAG

L 1 : GCTGGAGATTCAGGTTGCCAGATGGCTAGTTTGGCTGGTGCGAAGATCTG

L 2 : GCTGGAGATTCAGGTTGCCAGATGGCTAGTTTGGCTGGTGCGAAGATCTG

L 3 : GCTGGAGATTCAGGTTGCCAGATGGCTAGTTTGGCTGGTGCGAAGATCTG

L 4 : GCTGGAGATTCAGGTTGCCAGATGGCTAGTTTGGCTGGTGCGAAGATCTG

L10: GCTGGAGATTCAGGTTGCCAGATGGCTAGTTTGGCTGGTGCGAAGATCTG

L11: GCTGGAGATTCAGGTTGCCAGATGGCTAGTTTGGCTGGTGCGAAGATCTG

L 1 : CATAGGACTTTGTGCCTGGTCAAATGTTGTTGGCTGGATGGCGATTGCAC L 2 : CATAGGACTTTGTGCCTGGTCAAATGTTGTTGGCTGGATGGCGATTGCAC L 3 : CATAGGACTTTGTGCCTGGTCAAATGTTGTTGGCTGGATGGCGATTGCAC L 4 : CATAGGACTTTGTGCCTGGTCAAATGTTGTTGGCTGGATGGCGATTGCAC L10 : CATAGGACTTTGTGCCTGGTCAAATGTTGTTGGCTGGATGGCGATTGCAC L11 : CATAGGACTTTGTGCCTGGTCAAATGTTGTTGGCTGGATGGCGATTGCAC

L 1 : TTCTCTGGCCTAGCAATGGGCGCGACGAGCCACTGAGAGCGAATCAAGTG L 2 : TTCTCTGGCCTAGCAATGGGCGCGACGAGCCACTGAGAGCGAATCAAGTG L 3 : TTCTCTGGCCTAGCAATGGGCGCGACGAGCCACTGAGAGCGAATCAAGTG L 4: TTCTCTGGCCTAGCAATGGGCGCGACGAGCCACTGAAAGCGAATCAAGTG L10: TTCTCTGGCCTAGCAATGGGCGCGACGAGCCACTGAGAGCGAATCAAGTG L11: TTCTCTGGCCTAGCAATGGGCGCGACGAGCCACTGAGAGCGAATCAAGTG

L 1 : CGTGGgTGAAGTTTGCTTCAACAGTCTTATGGCTGGTGTTGTGAGCCCCG L 2 : CGTGGGTGAAGTTTGCTTCAACAGTCTTATGGCTGGTGTTGTGAGCCCCG L 3 : CGTGGGTGAAGTTTGCTTCAACAGTCTTATGGCTGGTGTTGTGAGCCCCG L 4 : CGTGGGTGAAGTTTGCTTCAACAGTCTTATGGCTGGGGTTGGGAGCCCCC L10 : CGTGGGTGAAGTTTGCTTCAACAGTCTTATGGCTGGTGTTGTGAGCCCCG L11 : CGTGGGTGAAGTTTGCTTCAACAGTCTTATGGCTGGTGTTGTGAGCCCCG 
Figure 1. (Continued)

L 1 : CGTTTTCTGGCTTCGATTTCGGTGGTCTTATGTATGGAGATAGGACAGAC 600

L 2 : CGTTTTCTGGCTTCGATTTCGGTGGTCTTATGTATGGAGATAGGACAGAC

L 3 : CGTTTTCTGGCTTCGATTTCGGTGGTCTTATGTATGGAGATAGGACAGAC

L 4 : CGTTTTCTGGCTTCAATTTCGGGGGTCCTTAAAAAATGAGATAGGGACAC

L10 : CGTTTTCTGGCTTCGATTTCGGTGGTCTTATGTATGGAGATAGGACAGAC

L11 : CGTTTTCTGGCTTCGATTTCGGTGGTCTTATGTATGGAGATAGGACAGAC

L 1: TCGTTTATAGCGAGTGCCGCTTTTGTGGCACTGTCTTTGTCCGACATCTG

L 2 : TCGTTTATAGCGAGTGCCGCTTTTGTGGCACTGTCTTTGTCCGACATCTG

L 3 : TCGTTTATAGCGAGTGCCGCTTTTGTGGCACTGTCTTTGTCCGACATCTG

L 4 : CACTCCAAAATAGCGGAGTGCCGGCCTTTGTTGGCCCTGTTCTTTTGTGC

L10 : TCGTTTATAGCGAGTGCCGCTTTTGTGGCACTGTCTTTGTCCGACATCTG

L11 : TCGTTTATAGCGAGTGCCGCTTTTGTGGCACTGTCTTTGTCCGACATCTG

L 1 : TCGCGAGTAGGTCGGTGGCCTCTCTGACCCGTCTTG 754

L 2 : TCGCGAGTAGGTCGGTGGCCTCTCTGACCCGTCTTG

L 3 : TCGCGAGTACGTCGGTGGCCTCTCTGACCCGTCTTG

L 4 : GTTGGCCCCTGGTTCTTTGTGGCGGGACATACGGG

L10 : TCGCGAGTAGGTCGGTGGCCTCTCTGACCCGTCTTG

L11 : TCGCGAGTAGGTCGGTGGCCTCTCTGACCCGTCTTG

Table 1. The distance between bases of sequence 6 species in the $28 \mathrm{~S}$ region of Lernaea

\begin{tabular}{ccccccccc}
\hline[ & $\mathbf{1}$ & $\mathbf{2}$ & $\mathbf{3}$ & $\mathbf{4}$ & $\mathbf{5}$ & $\mathbf{6}$ & $\mathbf{7}$ & $\mathbf{8}]$ \\
\hline$[1]$ & & {$[0.0]$} & {$[0.0]$} & {$[0.0]$} & {$[0.0]$} & {$[0.0]$} & {$[0.0]$} & {$[0.0]$} \\
{$[2]$} & 1.0 & & {$[0.0]$} & {$[0.0]$} & {$[0.0]$} & {$[0.0]$} & {$[0.0]$} & {$[0.1]$} \\
{$[3]$} & 1.0 & 1.0 & & {$[0.0]$} & {$[0.0]$} & {$[0.0]$} & {$[0.0]$} & {$[0.1]$} \\
{$[4]$} & 1.0 & 1.0 & 1.0 & & {$[0.0]$} & {$[0.0]$} & {$[0.0]$} & {$[0.1]$} \\
{$[5]$} & 0.1 & 0.1 & 0.4 & 0.1 & & {$[0.0]$} & {$[0.0]$} & {$[0.0]$} \\
{$[6]$} & 1.0 & 1.0 & 1.0 & 1.0 & 0.4 & & {$[0.0]$} & {$[0.1]$} \\
{$[7]$} & 1.0 & 1.0 & 1.0 & 1.0 & 0.1 & 1.0 & & {$[0.1]$} \\
{$[8]$} & 0.3 & 0.3 & 0.4 & 0.3 & 0.4 & 0.3 & 0.3 & \\
\hline
\end{tabular}

Note:

[1] \#Lernaea_cyprinaceae_G; [2] \#Lernaea_lophiara; [3] \#Lernaea_cyprinacea; [4] \#Lernaea_devastatrix [5] \#Lernaea_sp.; [6] \#Lernaea_oryzophila; [7] \#Lernaea_papuensis; [8] \#Dactilogyrus

Lernaea (Figure 2). First group were Lernaea cyprinacea G., L. papuensis, L. devastatrix, and L. Iophiara. Second group were Lernaea cyprinacea and L. oryzophilla, Lernaea sp. was in the third group. However, phylogenetic tree using neighbor joining (NJ) analysis found two groups among Lernaea (Figure 3). First groups were including Lernaea cyprinacea G., $L$. cyprinacea, L. papuensis, L. devastatrix, $L$. lophiara, and L. oryzophila; and second group was including Lernaea sp.

All species Lernaea were sequence on 28SrDNA region based on study by Song et al.
(2008) who found relationships among species in genus Ergasilidae (copepods) with Lernaea cyprinacea as an outgroup. The average percentage of codon $\mathrm{G}+\mathrm{C}$ was $51.6 \%$. Learnea cyprinacea G., Lernaea lophiara, and Lernaea papuensis had the same percentage of codon $\mathrm{G}+\mathrm{C}(51.7 \%)$. The percentage of $\mathrm{G}+\mathrm{C}$ codon for Lernaea cyprinacea is $51.6 \%$. Lernaea devastatrix, Lernaea sp., and Lernaea oryzophila are $51.8 \%, 51.3 \%$, and $51.5 \%$, respectively. There is no avalailable data sequence on Lernaea sp., except Lernaea cyprinacea in GenBank, to compare the result. 


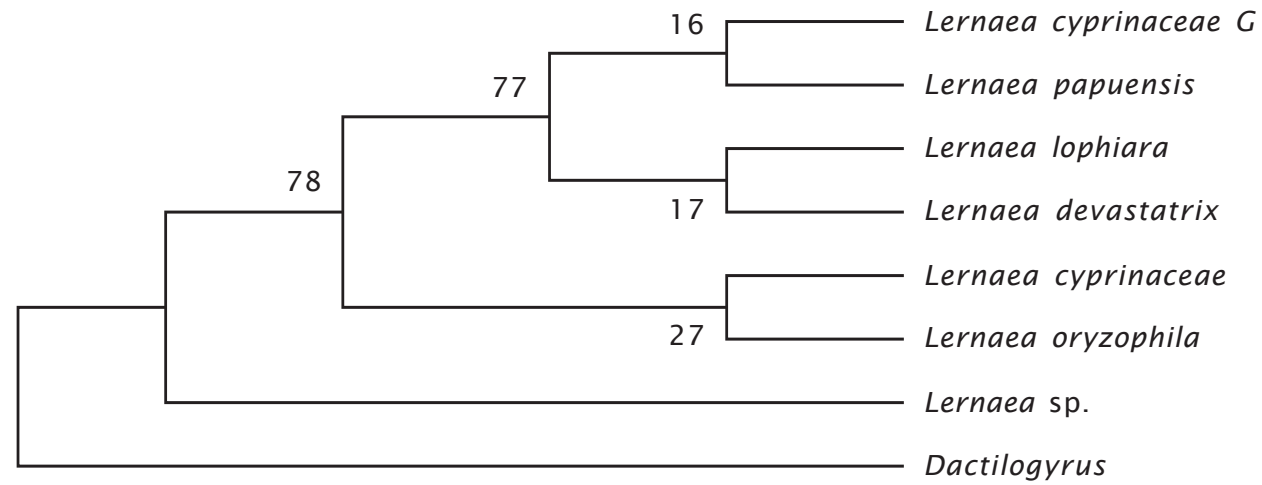

Figure 2. Phylogenetic tree from Lernaea sp. analyzed with maximum parsimony from sequence 6 species of Lernaea and L. cyprinacea GenBank with outgroups Dactilogyrus in $28 \mathrm{~S}$ region

Based on maximum parsimony (Figure 2) there are 3 clade among species of Lernaea. Clade I consists of Lernaea cyprinacea G., L. papuensis, L. Devastatrix, and L. lophiara. Clade II consist of Lernaea cyprinacea, and $L$. oryzophilla. Lernaea sp. is the only member of Clade III. Phylogenic relationship between clade I and clade II is closer than clade III. The closeness similarity among the species of Lernea is probably due to proximity of the historical relationship between Indonesia archipelago and Asian and Australian continents. Lernaea cyprinacea G., and L. papuensis; L. devastatrix, and L. lophiara; and L. cyprinacea and L. oryzophilla can be considered as sister taxon (sister). It means that they all share a common anchestor. Lernaea sp. can be re- garded as the begining of other species of Lernaea because it appears on the earliest in the phylogenetic tree. Lernaea cyprinacea G. and $L$. papuensis are the last descendant of Lernaea sp.

However, there are only two clade among species Lernaea based on neighbor joining tree (NJ) method (Figure 3). Clade I consists of L. cyprinacea GenBank, L. cyprinacea, L. oryzophila, $L$. lophiara, $L$. devastarix, and $L$. papuensis. Clade II is Lernaea sp. From the obtained result we concluded that the species Lernaea is monophyletic. Ho (1998) stated that Lernaeidae, Ozmanidae, and Ascidicolida are monophyletic group, which are derived from a common ancestor.

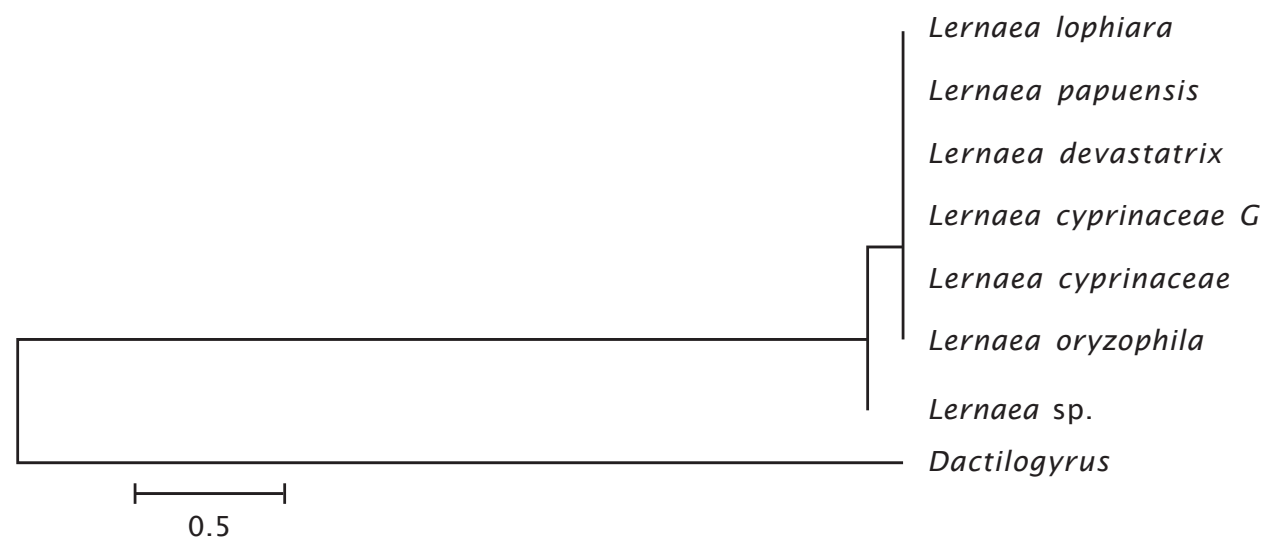

Figure 3. Phylogenetic tree from Lernaea sp. analyzed with neighbor joining from sequence 6 species of Lernaea and L. cyprinacea GenBank with outgroups Dactilogyrus in $28 \mathrm{~S}$ region 
The evolution of Lernaea mainly associated with the development and modification of the holdfast organ. The final form of the holdfast organ is determined by the consistency of fish tissues by which a parasitic animal attaches itself to its host. So many variations found in holdfast's shape. Strong influence of environment on morphology of Lernaea expressed by Poddubnaja (1974) in Kabata (1985) who found L. cyprinacea, L. ctenopharyngdonis, L. quadrinucifera adult females origates from the same individual eggs. So far, there is no studies on genetic molecular of Lernaea sp. This study perhaps is the iniatial study of molecular Lernaea.

\section{CONCLUSION}

Phylogenetic tree from maximum parsimony (MP) and neighbor joining (NJ) based on sequence in $28 \mathrm{~S}$ rDNA do not show differences among species Lernaea. There are 3 variation groups of Lernaea from arwana Irian fish in Indonesia based on maximum parsimony. Lernaea papuensis, $L$. devastatrix, and $L$. lophiara are in one group; L. cyprinacea and $L$. oryzophilla are in one group; and Lernaea sp is in different group. However, based on neighbour joining there are 2 groups variation of Lernaea from Arwana Irian, ie. Lernaea papuensis, L. devastatrix, L. lophiara, L. cyprinacea, and L. oryzophilla are in one group; and Lernaea sp. is in different group. This indicates that phylogenetic reconstructions should be in other region, such as in the ITS region. This result is also suggested that Lernaea was monophyletic.

\section{REFERENCES}

Berry, C.R., Babey, G.J., \& Shrader, T. 1991. Effect of Lernaea cyprinacea (Crustacea: Copepoda) on stocked rainbow trout (Onchorhynchus mykiss). J. of Wildlife Dis., 27(2): 206-213.

Boxshall, G.A. 1981. A new spesies of Lernaea (Copepoda: Cyclopoida) from Papua-New Guinea. Bulletin British Museum History, 40: 117-120.

Boxshall, G.A., Montu, M.A., \& Schwarzbold, A. 1997. A new species of Lernaea L (Copepoda: Cyclopoida) from Brazil, with notes on its ontogeny. Systematic Parasitology, 37: 195-205.

Ho, J.S. 1998. Cladistics of The Lernaeidae (Cyclopoida), A Major Family of Freshwater Fish Parasites. J. of Marine System, 15: 177183.

Kabata, Z. 1985. Parasites and diseases of fish cultured in the tropics. Taylor and Francis. Philadelphia, $318 \mathrm{pp}$.

Kumar, S., Tamura, K., \& Nei, M. 2004. MEGA3: Integrated Software for Molecular Evolutionary Genetics Analysis and Sequence Alignment. Brief Bioinform, 5: 150-163.

Kularatne, M., Subangsinghe, R.P., \& Shariff, M. 1994. Investigations on the lack of aqcuired immunity by the Javanese carp, Punctius gonionotus (Bleeker), against the crustacean parasite, Lernaea minuta (Kuang). J. Fish \& Shellfish Imun., 4: 107114.

Marina, H., Beatty, S.J., Morgan, D.L., Doupe, R.G., \& Lymbery, A.J. 2008. An introduced parasite, Lernaea cyprinacea L., found on native freshwater fishes in the South West of Western Australia. J. of the Royal Soc.of Westen Australia, 91: 149-153.

Paperna, I. 1996. Parasites, infections and diseases of fishes in Africa : an update. Israel.EIFAA Technical Paper - CIFA/T31.

Shariff, M., Kabata, Z., \& Sommerville, C. 1986. Host susceptibility to Lernaea cyprinacea L. and its treatment in a large aquarium system. J. of Fish Dis., 9: 393-401.

Song, Y., Wang, G.T., Yao, W.J., Gao, G., \& Nie, P. 2008. Phylogeny of freshwater parasitic copepods in the Ergasilidae (Copepoda : Poecilostomatoida) based on $18 \mathrm{~S}$ and $28 \mathrm{~S}$ rDNA sequences. J. Parasitol, 102: 299306.

Tasawar, Z., Hanif, M., Lashari, M.H., \& Hayat, C.S. 2007. The prevalence of lernaeid ectoparasites in Mori (Cirrhinus migala) fish. J. Pakistan Vet., 27(4): 176-178.

Thompson, J. D., Higgins, D.G., \& Gibson, T.J. 1994. CLUSTAL W: improving the sensitivity of progressive multiple sequence alignment through sequence weighting, position-specific gap penalties and weight matrix choice. Nucleic Acids Res., 22: 4,6734,680 . 